\title{
Amorphous Solar Modules Simulation and Experimental Results: Effect of Shading
}

\author{
Luis Fialho $^{1,3}$, Rui Melício ${ }^{1,3}$, Victor M.F. Mendes ${ }^{1,2}$, João Figueiredo ${ }^{1,3}$, \\ and Manuel Collares-Pereira ${ }^{1}$ \\ ${ }^{1}$ Universidade de Évora, Évora, Portugal \\ ruimelicio@uevora.pt \\ ${ }^{2}$ Instituto Superior of Engenharia de Lisboa, Lisbon, Portugal \\ ${ }^{3}$ IDMEC/LAETA, Instituto Superior Técnico, Universidade de Lisboa, Lisbon, Portugal
}

\begin{abstract}
This paper focuses on the modeling of PV systems by the five parameters model, consisting on a current controlled generator, single-diode, a shunt and series resistances. An assessment for the identification of the parameters is used requiring data on open circuit, maximum power and short circuit tests. A simulation of a photovoltaic system on a parallel of two series connected amorphous solar modules under the effect of partial shading is presented. The estimated parameters are validated by a comparison with experimental measurements on photovoltaic modules.
\end{abstract}

Keywords: PV system, effect of shading, algorithm for parameter estimation, simulation, experimental results, shading on amorphous PV.

\section{Introduction}

The demand for energy, the foreseeable future scarcity and the price of fossil fuels coupled with the need for carbon footprint reduction turn out a political consciousness of the importance of energy savings and energy efficiency usage [1] and programs on the Demand-side Management have been developed in order to assist consumers on energy usage. Moreover, alternative sources of energy, for instance, wind and solar energy sources have turn out to be attractive for exploitation, not only for large scale systems, but also for micro and mini scale conversion systems [2], Disperse Generation owned by consumer. Disperse Generation significantly utilizes solar energy as a primary source of energy and have came into sight the utilization of Thermal and Photovoltaic (PV) systems. A PV system directly converts solar energy into electric energy. The main device of a PV system is a solar cell. Cells may be grouped to form arrays and panels. A PV array may be either a panel or a set of panels connected in series or parallel to form large PV systems without or with tracking systems in order to achieve higher values of energy conversion during sunny days due to the diverse perpendicular positions to collect the irradiation from the sun. The performance of a PV array depends on the operating conditions especially on solar irradiation, temperature, array configuration and shading. The shading on a PV array, for instances, due to a passing cloud or neighboring buildings causes not only power losses, but also further non-linear effects on the array V-I characteristics [3]. In order 
to protect the cells from destructive reverse voltages in case of shadowing or other abnormalities, a bypass diodes are utilized, for example, one bypass diode connected in parallel with each set of 18 cells [4] or with a panel is common practice as a compromise between protection and increase on the cost due to the extra pn-junction.

The impact of the shadow is important in case of large PV power system installations. Under partially shaded conditions, the PV characteristics of the system acquire complexity, appearing multiple peaks. Therefore, the importance to understand and to predict the shadow consequences in order not only to extract energy at the maximum possible power [3], but also to assess the protection on the systems.

\section{Relationship to Collective Awareness Systems}

Current trend in energy supply and usage are perceptibly economic, environmental and social unsustainable. There is a growing collective awareness of the urgent need to turn political statements and analytical work about the present unsustainable trend in energy supply and usage into concrete action in order to achieve what is anticipated as a sustainable development. The development of collective awareness platforms for a sustainable development allows the advancement not only of collective decisionmaking tools, but also of innovation mechanisms. These platforms allow individuals and groups to effectively react to environmental sustainability challenges, with an effective awareness of problems and possible solutions [5]. Solar energy is the most abundant form of energy on earth. PV systems are expanding due to supporting policies and cost reductions. Hence, PV systems are commercially available and a reliable technology with a potential for usage growth in almost everywhere. Although, can be argued that PV systems usage is not relevant in nowadays, PV systems are expected to be of relevant usage over the next decades [6].

\section{$3 \quad$ Modeling}

The equivalent circuit model used in this paper is the five parameters equivalent circuit, consisting in a current controlled generator, a single-diode, a shunt and series resistances as shown in Fig. 1.

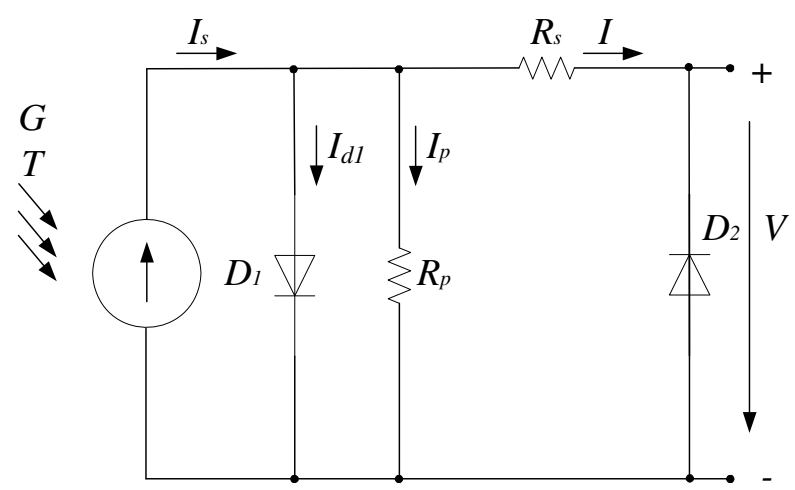

Fig. 1. Equivalent circuit of the solar module 
In Fig.1, although not a part of the equivalent circuit, the anti-parallel bypass diode $D_{2}$ is shown for connection illustration purpose. $G$ is the solar irradiance, $T$ is the cell $p-n$ junction temperature in $[\mathrm{K}], R_{p}$ is the equivalent shunt resistance, $R_{s}$ is the equivalent series resistance, $I$ is the output current, $V$ is the output voltage, $I_{S}$ is the photo generated electric current, $I_{d 1}$ is the current at diode $D_{1}, I_{p}$ is the leakage current. If cells are associated in a module, an array or a panel and are equally subjected to the same irradiance, the junctions are at the same temperature $T$, then the circuit in Fig. 1 is also the equivalent circuit for the association, but with a suitable transformation for the parameters. The thermal voltage of a solar cell $V_{T}$ is given by:

$$
V_{T}=k T / q
$$

where $k$ is the Boltzman's constant, $q$ is the electron charge.

The I-V characteristic is given by the implicit function associated with the model shown in Fig. 1 for the solar cell and is given by:

$$
I=I_{S}-I_{0}\left(e^{\frac{V+I R_{S}}{m V_{T}}}-1\right)-\frac{V+I R_{S}}{R_{p}}
$$

where $I_{0}$ is the diode reverse bias saturation current, and $m$ is the diode ideality factor. (2). This equation has to be solved by an iterative method to determine for instance the output current in function of the output voltage. From (2), considering the short-circuit condition $I_{S}$ is given by:

$$
I_{S}=I_{S C}\left(1+\frac{R_{s}}{R_{p}}\right)+I_{0}\left(e^{\frac{I_{s c} R_{s}}{m V_{T}}}-1\right)
$$

The second term of (3) is usually small in comparison with the first term [7], [8]. So, the usual approximation considered for $I_{S}$ is given by:

$$
I_{S} \approx I_{S C}\left(1+\frac{R_{s}}{R_{p}}\right)
$$

Also from (2), considering the open circuit condition and due to the fact that the exponential at this condition is significant greater than one, $I_{S}$ is given by:

$$
I_{S} \approx I_{O} e^{\frac{V_{o c}}{m V_{T}}}+\frac{V_{o c}}{R_{p}}
$$

Equating (4) to (5), $I_{0}$ is approximated by the expression given by:

$$
I_{O} \approx\left[I_{S C}\left(1+\frac{R_{s}}{R_{p}}\right)-\frac{V_{o c}}{R_{p}}\right] e^{-\frac{V_{o c}}{m V_{T}}}
$$


Hence, $I_{0}$ can be computed by (6), if the parameters $R_{p}, R_{s}, T, m$ are known.

Substituting (4) into (2) at Maximum Power Point (MPP) condition and due to the fact that the exponential at this condition is significant greater than one, $I_{0}$ is approximated by the expression given by:

$$
I_{0} \approx\left[\left(I_{S C}-I_{M P}\right)\left(1+\frac{R_{s}}{R_{p}}\right)-\frac{V_{M P}}{R_{p}}\right] e^{-\frac{V_{M P}+I_{M P} R_{s}}{m V_{T}}}
$$

The electric power output equation and (2) allow for the determination of the derivative of the $I$ in order to $V$ at MPP, respectively given by:

$$
\left(\frac{\partial I}{\partial V}\right)_{M P}=-\frac{I_{M P}}{V_{M P}}, \quad\left(\frac{\partial I}{\partial V}\right)_{M P}=-\frac{V_{M P}-I_{M P} R_{s}}{V_{M P}}\left(\frac{I_{0}}{m V_{T}} e^{\frac{V_{M P}+I_{M P} R_{s}}{m V_{T}}}+\frac{1}{R_{p}}\right)
$$

Considering (7) and (8) $I_{M P}$ is approximated by the implicit expression given by:

$$
I_{M P} \approx \frac{\left(V_{M P}-I_{M P} R_{s}\right)}{m V_{T}}\left[\left(I_{S C}-I_{M P}\right)\left(1+\frac{R_{s}}{R_{p}}\right)-\frac{V_{M P}-m V_{T}}{R_{p}}\right]
$$

Assuming (9) as having enough accurateness [8] for the parameter estimation $R_{p}$ is approximated by the expression given by:

$$
R_{p} \approx \frac{V_{M P}-I_{M P} R_{s}-m V_{T}}{\left(V_{M P}-I_{M P} R_{s}\right)\left(I_{s c} / I_{M P}-1\right)-m V_{T}} \frac{V_{M P}}{I_{M P}}-R_{s}
$$

and $R_{s}$ from (6) of [8] is given by:

$$
R_{s} \approx \frac{V_{o c}-V_{M P}}{I_{M P}}-\frac{m V_{T}}{I_{M P}} \ln \left[\frac{V_{M P}-I_{M P} R_{s}+m V_{T}}{m V_{T}} \frac{I_{s c}\left(R_{s}+R_{p}\right)-V_{o c}}{I_{s c}\left(R_{s}+R_{p}\right)-2 V_{M P}}\right]
$$

The ideality factor can be evaluated by (2) at maximum power point, replacing $I_{s}$ given by (4), $I_{0}$ given by (6) and taking logarithms [8]. Hence $m$ is given by:

$$
m \approx \frac{1}{V_{T}} \frac{V_{o c}-V_{M P}-I_{M P} R_{s}}{\ln \left[I_{s c}\left(R_{s}+R_{p}\right)-V_{o c}\right]-\ln \left[\left(I_{s c}-I_{M P}\right)\left(R_{s}+R_{p}\right)-V_{M P}\right]}
$$

But $R_{s}>0$, then (11) imposes the implicit relationship given by:

$$
m<\frac{V_{o c}-V_{M P}}{V_{T} \ln \left[\frac{V_{M P}-I_{M P} R_{s}+m V_{T}}{m V_{T}} \frac{I_{s c}\left(R_{s}+R_{p}\right)-V_{o c}}{I_{s c}\left(R_{s}+R_{p}\right)-2 V_{M P}}\right]}
$$

Also to ensure that $R_{p}<\infty$, then the denominator in (10) imposes the relationship given by:

$$
m<\frac{\left(V_{M P}-I_{M P} R_{s}\right)\left(I_{s c} / I_{M P}-1\right)}{V_{T}}
$$


The above expressions are capable of giving values for the five parameters equivalent circuit of a solar cell, using only data from open circuit, maximum power and short circuit tests. A Matlab/Simulink coding was carried out based on those expressions in order to simulate the behavior of PV systems.

\section{Simulation}

A simulation study concerning with the data measured from PV silicon amorphous solar modules Kaneka KA58 provided in [9] and placed in a photovoltaic facility at the Laboratório Nacional de Energia e Geologia (LNEG) in Lisbon, Portugal, is presented for illustration purpose. The coordinates for the PV modules site are: 3846'18.50'’'N, 9॰10'38.50'’ W.

The data for the silicon amorphous solar module Kaneka KA58 at STC [10] are shown in Table 1.

Table 1. Data for the Kaneka KA58 solar module at STC

\begin{tabular}{ccccccc}
\hline Technology & $V_{m}^{*}$ & $I_{m}^{*}$ & $V_{o c}^{*}$ & $I_{s c}^{*}$ & $\beta_{o c}$ & $\alpha_{s c}$ \\
\hline Amorphous & $63 \mathrm{~V}$ & $0.92 \mathrm{~A}$ & $85 \mathrm{~V}$ & $1.12 \mathrm{~A}$ & $-206 \mathrm{mV} /{ }^{\circ} \mathrm{C}$ & $1.3 \mathrm{~mA} /{ }^{\circ} \mathrm{C}$ \\
\hline
\end{tabular}

The characteristic curve for each tested PV module is measured outdoors quasisimultaneously with the measurement of the reference unit I-V curve. The I-V curve is then converted to STC conditions by using the procedure described in IEC 60891 [11]. The I-V curve and the tracer used is shown in Fig. 2.

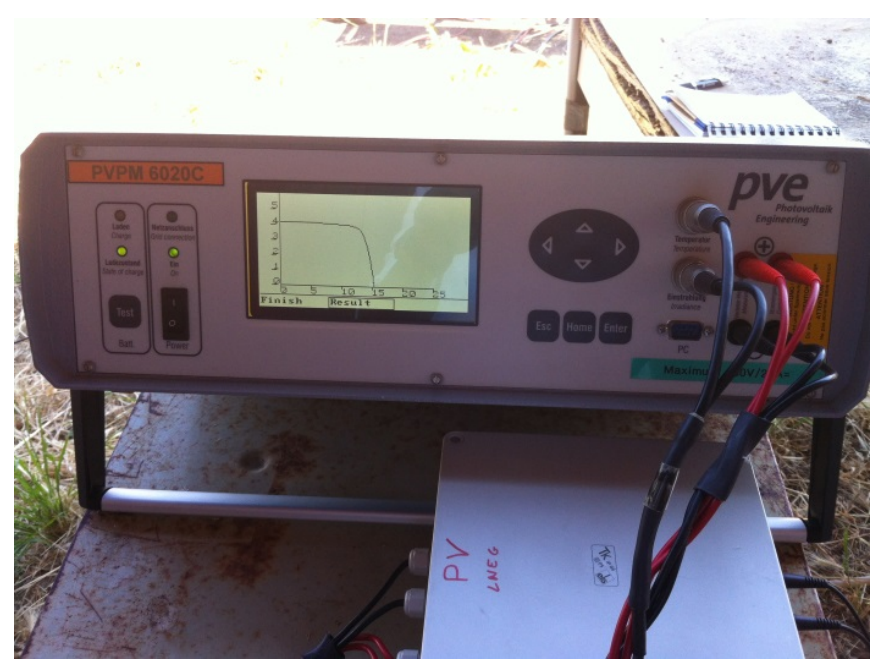

Fig. 2. Experimental I-V curves 
The simulation results are compared with experimental observation carried out for an amorphous PV module technology with two modules connected in series.

The I-V curves simulated and experimental without shading are shown in Fig. 3.

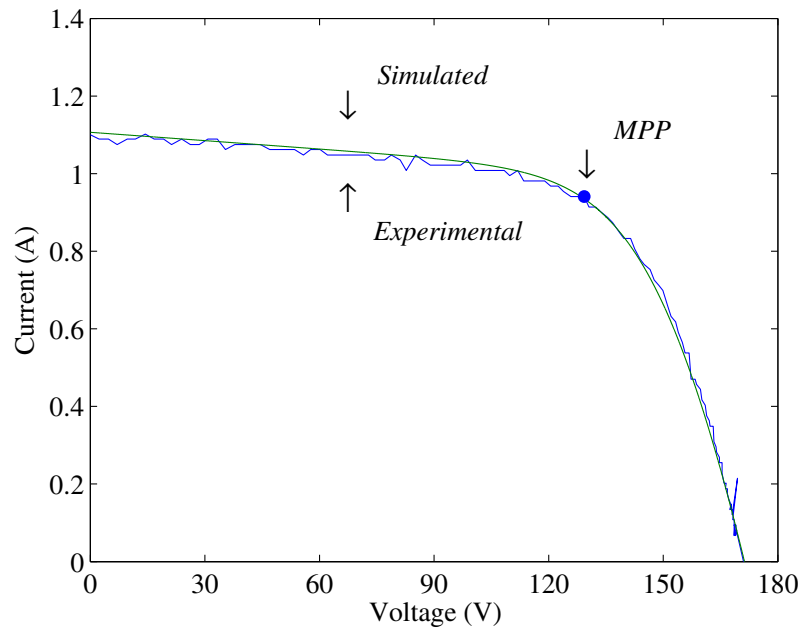

Fig. 3. I-V curves simulated and the experimental

The partial shading simulation was carried with twelve PV modules associating in parallel two series of six modules. The configuration in study with partial shading is shown in Fig. 4.

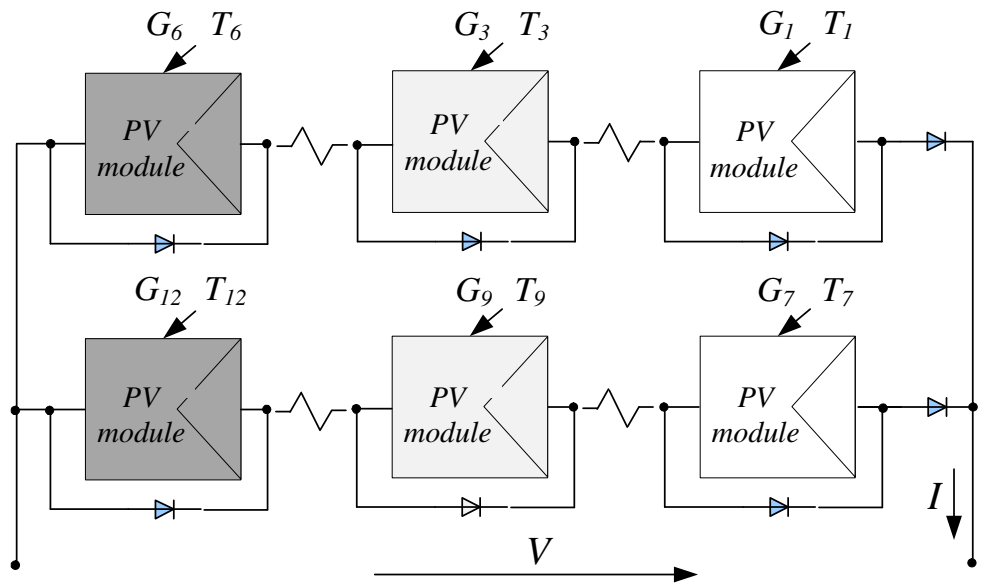

Fig. 4. Configuration for the simulation with partial shading 
In Fig. 4, the partial shading is given by the following condition: $G=1200 \mathrm{~W} / \mathrm{m}^{2}$ for the modules $\{1,2,4,5,7,8,10,11\} ; G=1000 \mathrm{~W} / \mathrm{m}^{2}$ for the modules $\{3,9\}$ and $G=700 \mathrm{~W} / \mathrm{m}^{2}$ for the modules $\{6,12\}$. The I-V and P-V curves obtained by simulation of partial shading are respectively shown in Fig. 5 and Fig. 6.

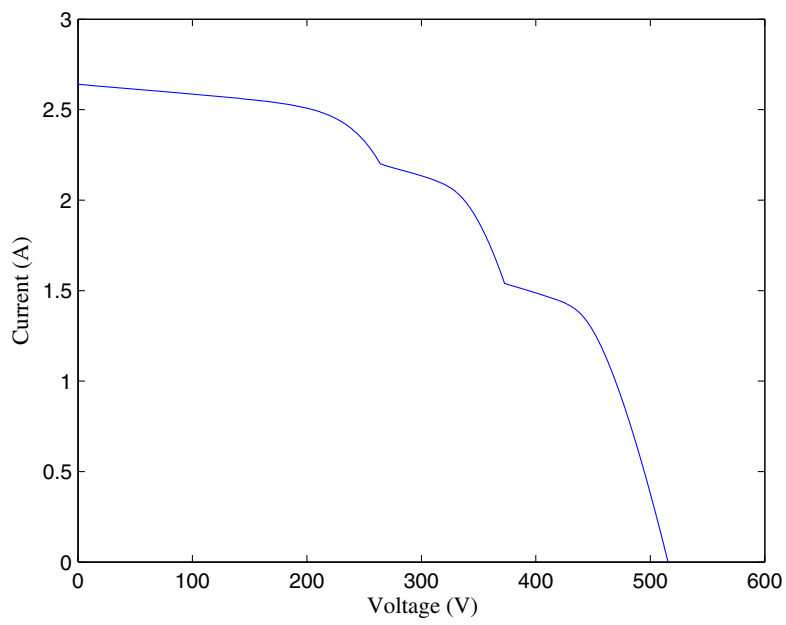

Fig. 5. I-V curve simulated for the PV modules with partial shading

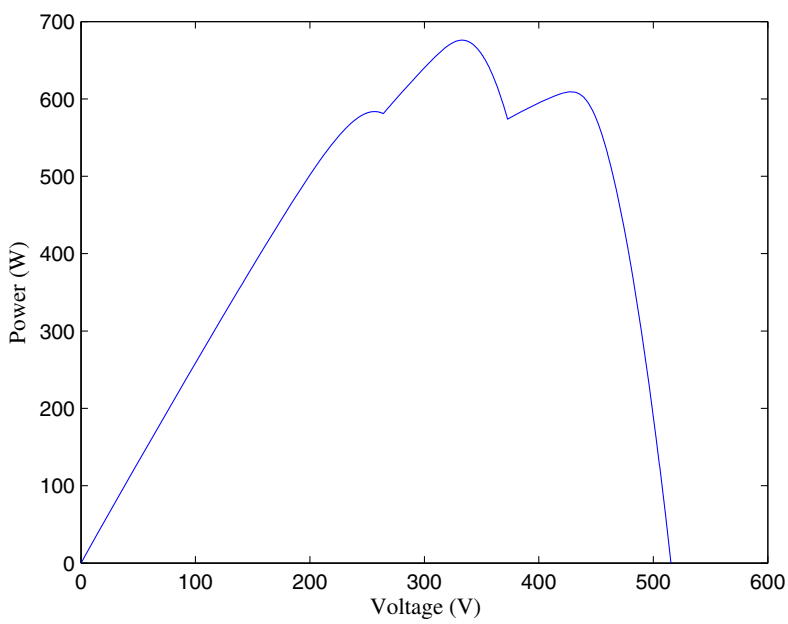

Fig. 6. $P_{-} V$ curve simulated for the PV modules with partial shading 
Fig. 5 and Fig. 6 reveal the implication in the I-V and P-V curves due to the shading, i.e., due to the non-uniform illumination on the modules there are local MPPs, which can pose a difficult to the MPP tracking (MPPT) system and there is a loss on the expected energy conversion.

\section{Conclusions}

The five parameters solar cell equivalent circuit is used in this paper in order to acquire analytically I-V curves. The five parameters are estimated by a method using a set of equations and requiring information on open circuit, maximum power and short circuit tests. A simulation study using the five parameters estimation is reported for illustration purpose, presenting the simulate performance of a PV system before installation in sitú in what regard shading conditions on a parallel of two series connected amorphous solar PV modules technology, assessing the performance given by the I-V and P-V curves due to a partial shading simulation. The shading curves show multiple local MPPs, which can pose a difficult to the MPPT algorithm. The knowledge of these curves can be useful to minimize power losses and avoid damage to the solar cells due to hot spots during high power losses. This shading simulation is handy to help the designer in what regards extracting the maximum energy and protection of the system. A comparison between simulated and the experimental results shows that the estimation is a satisfactory approximation method.

Acknowledgments. This work was partially supported by Fundação para a Ciência e a Tecnologia, through IDMEC under LAETA, Instituto Superior Técnico, Universidade de Lisboa, Portugal. The authors gratefully acknowledge to Ms. L. Giacobbe for providind the measured data of amorphous PV modules at LNEG.

\section{References}

1. Seixas, M., Melicio, R., Mendes, V.M.F.: A simulation for acceptance of two-level converters in wind energy systems. In: Proc. of JIUE 2013 - $3^{\text {a }}$ Jornadas de Informática da Universidade de Évora, Évora, Portugal, pp. 75-79 (2013)

2. Blaabjerg, F., Chen, Z., Kjaer, S.B.: Power electronics as efficient interface in dispersed power generation Systems. IEEE Trans. Power Electron 19(5), 1184-1194 (2004)

3. Patel, H., Agarwal, V.: MATLAB-based modeling to study the effects of partial shading on PV array characteristics. IEEE Trans. Energy Conversion 23(1), 302-310 (2008)

4. Quaschning, V., Hanitsch, R.: Numerical Simulation of Photovoltaic Generators with Shaded Cells. In: 30th Universities Power Engineering Conference, London, UK, pp. 583-586 (1995)

5. European Commission: Collective Awareness Platforms for Sustainability and Social Innovation. Europe 2020 Initiative, retrieved (2013),

http: / / ec . europa.eu/digital-agenda/en/collective-awarenessplatforms-sustainability-and-social-innovation

6. International Energy Agency: Technology roadmap, solar photovoltaic energy (2010)

7. Carrero, C., Rodríguez, J., Ramírez, D., Platero, C.: Simple estimation of PV modules loss resistances for low error modelling. Renewable Energy 35, 1103-1108 (2010) 
8. Carrero, C., Rodríguez, J., Ramírez, D., Platero, C.: Accurate and fast convergence method for parameter estimation of PV generators based on three main points of the I-V curve. Renewable Energy 36, 2972-2977 (2011)

9. Giacobbe, L.: Validação de modelos matemáticos de componentes de sistemas fotovoltaicos. Master Thesis, DEEC/IST (2005) (in Portuguese)

10. Kaneka Photovoltaic Products Information, http://www.pv . kaneka.co.jp

11. IEC 60891: Procedures for temperature and irradiance corrections to measured I-V characteristics of crystalline silicon photovoltaic devices (2009) 